\title{
Inconclusive abdominal CT findings in pediatric patients with appendicitis: lessons from negative appendectomy cases
}

This article was published in the following Dove Press journal:

Reports in Medical Imaging

23 March 2016

Number of times this article has been viewed

\section{Younglim Kim \\ Suk-Bae Moon}

Department of Surgery, Kangwon National University Hospital, Kangwon National University Graduate School of Medicine, Chuncheon, South Korea
Correspondence: Suk-Bae Moon Department of Surgery, Kangwon National University Hospital,

Kangwon National University Graduate

School of Medicine, Chuncheon 200-722,

South Korea

Tel +82332589209

Fax +82332582169

Email sukbae75.moon@gmail.com
Purpose: Although abdominal computed tomography (ACT) has high sensitivity and specificity in helping to detect appendicitis in pediatric patients, diagnostic challenges still exist for some cases despite undergoing ACT, and negative appendectomies still persist. The aim of this study was to elucidate some ACT findings that support true appendicitis for patients who had inconclusive preoperative ACT findings.

Patients and methods: For the 49 patients whose preoperative ACT diagnoses were inconclusive (ten negative appendicitis; 39 true appendicitis), the ACT findings were retrospectively measured for the following features: appendiceal enlargement, presence of appendicolith or hyperdense materials in the appendix, periappendiceal fat inflammation, periappendiceal fluid collection, appendiceal wall thickening, appendiceal gas, and right lower quadrant lymphadenopathy. These features were compared between true appendicitis and negative appendicitis cases.

Results: The presence of an enlarged appendix of diameter $>8 \mathrm{~mm}$ or periappendiceal fat inflammation strongly correlated with true appendicitis, while the absence of appendiceal wall thickening or mesenteric lymphadenopathy correlated with negative appendicitis.

Conclusion: If the ACT findings were inconclusive for patients suspected of having appendicitis, factors such as periappendiceal fat inflammation, an enlarged appendix, appendiceal wall thickening, and mesenteric lymphadenopathy should be cautiously assessed to discriminate true appendicitis from negative appendicitis.

Keywords: inconclusive abdominal CT, acute appendicitis, periappendiceal fat inflammation, appendiceal enlargement, wall thickening, lymphadenopathy

\section{Introduction}

Acute appendicitis is one of the most common causes of an acute abdomen requiring surgical intervention in children. Traditionally, because of concerns regarding radiation hazards, ultrasonography (USG) has been the usual modality of choice for radiological diagnosis of pediatric patients suspected of having appendicitis. Currently, because of its convenience, readiness, and diagnostic accuracy, abdominal computed tomography (ACT) is increasingly utilized to help diagnose acute appendicitis in pediatric emergency departments. ${ }^{1,2}$ Although ACT has high sensitivity and specificity in the detection of appendicitis in pediatric patients, diagnostic challenges still exist for patients who have undergone ACT, and a negative appendectomy is sometimes unavoidable in such cases; the current negative appendectomy rate is $\leq 10 \%$. 3,4

On the basis of ACT findings, Stengel et $\mathrm{al}^{5}$ classified the likelihood of appendicitis into five grades: grade 1, definitely not appendicitis; grade 2, nonvisualization of appendix with no secondary signs of inflammation; grade 3, equivocal; grade 4, 
probable; and grade 5, high possibility of or compatible with appendicitis. Using this classification, only grade 1 or 5 helps provide a definitive conclusion for surgeons, and the remaining grades suggest weaker or stronger clues regarding appendicitis; thus, the final radiological diagnosis remains inconclusive.

In this study, to avoid unnecessary negative appendectomies, we aimed to elucidate some ACT findings that support true appendicitis for patients who had inconclusive preoperative ACT findings regarding a definite diagnosis by comparing the ACT findings between pathological true appendicitis and negative appendicitis cases.

\section{Patients and methods}

\section{Patients}

The medical records of a total of 400 pediatric patients who underwent an operation for acute appendicitis between January 2007 and December 2014 were retrospectively reviewed. Of these patients, we excluded the following patients: patients who had undergone only USG $(n=58)$ or had no imaging evaluation ( $\mathrm{n}=29$ ) before surgery, and those who had atypical appendicitis (tuberculous appendicitis, $n=1$ ). Among the patients who had undergone ACT before surgery, patients for whom a definitive diagnostic conclusion (defined as Stengel grade 1 or 5 according to Stengel's ${ }^{5}$ classification) could be obtained were excluded $(n=263)$ after reviewing the original radiological reports, and inconclusive cases (defined as Stengel grade 2, 3, or 4) were enrolled in the final analysis ( $n=49$, study group). According to the pathological reports, 49 patients in the study group were further divided into a true appendicitis group $(n=39)$ and a negative appendicitis group $(n=10)$.

\section{Assessment of ACT findings}

For the 49 patients in the study group, the ACT findings were retrospectively assessed for the following features: appendiceal enlargement, presence of an appendicolith or hyperdense materials in the appendix, periappendiceal fat inflammation, periappendiceal fluid collection, appendiceal wall thickening, appendiceal gas, and right lower quadrant lymphadenopathy. The diameter of the appendix was measured from the outer to outer walls, and the appendix was considered enlarged if the diameter was $>8 \mathrm{~mm} .{ }^{6}$ Appendicolith was defined as an intraluminal lesion that demonstrated a high density, a density similar to that of the adjacent bone. ${ }^{7}$ Hyperdense material in the appendix was defined as an intra-appendiceal lesion that showed up as a hyperdense lesion as compared with the adjacent bowel wall. Wall thickness was measured from the luminal surface to the serosal layer and was considered to be thickened if it measured $>2.2 \mathrm{~mm} .{ }^{8}$ Lymphadenopathy was defined as an enlarged node measuring $>8 \mathrm{~mm}$ at its smallest diameter. ${ }^{9}$

\section{Statistical methods}

All the measured features were interpreted as dichotomous variables. Statistical analyses were performed using SPSS software, Version 18.0 (SPSS Inc., Chicago, IL, USA). Dichotomous variables were compared using the Student's $\chi^{2}$ test between the true appendicitis and negative appendicitis groups, and significance was assigned at a $P$-value of $<0.05$.

\section{Ethics statements}

This study was approved by the Kangwon National University Hospital Institutional Review Board (KNUH-2015-08-003). This review was conducted in accordance with all guidelines stipulated by the Declaration of Helsinki. Because no interventional experimentation whatsoever was involved and the study was retrospective, the Kangwon National University Hospital Institutional Review Board decided that written patient consent was not required.

\section{Results}

\section{Patient demographics}

During the observation period, 400 patients underwent an appendectomy, and of these patients, 46 were considered negative appendectomy cases; thus, the overall negative appendectomy rate in our institute was $11.5 \%$. In the study group $(n=49)$, ten patients had a negative appendectomy, and the negative appendectomy rate was $20.4 \%$. There were no statistical differences between the negative and true appendicitis groups in terms of age and sex ratio. One patient in the negative appendicitis group underwent a complementary USG after ACT, and one patient in the true appendicitis group underwent ACT after an inconclusive USG. Most of the cases had simple appendicitis in the true appendicitis group, and in the absence of histological evidence of appendicitis, various conditions were observed, such as lymphoid hyperplasia or serosal congestion, in the negative appendicitis group (Table 1).

\section{ACT findings}

The mean maximal diameter of the appendix was $9.5 \mathrm{~mm}$ in the true appendicitis group and $6.7 \mathrm{~mm}$ in the negative appendicitis group, and according to the definition of appendiceal enlargement ( $>8 \mathrm{~mm}$ in maximal diameter), only one case 
Table I Patient demographics

\begin{tabular}{|c|c|c|}
\hline Patient & $\begin{array}{l}\text { Negative appendicitis } \\
(\mathrm{N}=10)\end{array}$ & $\begin{array}{l}\text { True appendicitis } \\
(\mathrm{N}=39)\end{array}$ \\
\hline Mean age (years) & 14.2 (range: 9-18) & I3.6 (range: 6-18) \\
\hline Male:female ratio & $5: 5$ & $22: 17$ \\
\hline \multirow[t]{2}{*}{ Imaging modalities } & 9 ACT only & 38 ACT only \\
\hline & $\mathrm{I}$ ACT $\rightarrow$ USG & I USG $\rightarrow$ ACT \\
\hline Pathological & 2 Lymphoid hyperplasia & 9 Exudative appendicitis \\
\hline \multirow[t]{3}{*}{ diagnosis } & 2 Active serositis & $\begin{array}{l}30 \text { Suppurative } \\
\text { appendicitis }\end{array}$ \\
\hline & 2 Serosal congestion & $\begin{array}{l}2 \text { Gangrenous } \\
\text { appendicitis }\end{array}$ \\
\hline & $4 \mathrm{NDA}$ & \\
\hline
\end{tabular}

Abbreviations: ACT, abdominal computed tomography; NDA, no diagnostic abnormalities; USG, ultrasonography.

$(10 \%)$ had an enlarged appendix in the negative appendicitis group, while there were 29 cases $(74.4 \%)$ in the true appendicitis group; the difference was statistically significant $(P=0.000)$. Periappendiceal fat inflammation or fat stranding was observed in only one patient (10\%) in the negative appendicitis group and 32 patients $(82.1 \%)$ in the true appendicitis group $(P=0.000)$. Appendiceal wall thickening was observed in all patients $(100 \%)$ in the true appendicitis group and in six patients (Figure 1,60\%) in the negative appendicitis group ( $P=0.001)$. Mesenteric lymphadenopathy was observed in 38 patients (97.4\%) in the true appendicitis group and six patients (Figure 2, 60\%) in the negative appendicitis group $(P=0.004)$. For the other measured findings, there were no significant differences between the two groups. The ACT findings are summarized in Table 2, along with their odds ratios and $95 \%$ confidence intervals.

\section{Discussion}

In the past, acute appendicitis was a disease diagnosed using physical examinations and laboratory tests, and a negative appendectomy rate of $\leq 40 \%$ was acceptable for children. ${ }^{10,11}$ Today, the negative appendectomy rate has greatly decreased because of advancements in USG and $\mathrm{ACT}$, but inconclusive cases still occur; the current negative appendectomy rate is $<10 \% .^{12}$ The aim of this study was to elucidate ACT findings that would be helpful in differentiating between true and negative appendicitis for inconclusive cases after ACT.

During the study period, the overall negative appendectomy rate in our institute was $11.6 \%$, which was similar to or slightly higher than that of other studies. ${ }^{12}$ However, it jumped to $20.4 \%$ in the ACT-inconclusive patients in the study group, implying that a more refined interpretation of ACT findings is necessitated for suspected appendicitis in children. The ACT diagnosis of acute appendicitis has usually been based on the visualization of an appendix $>6 \mathrm{~mm}$ in maximal diameter, with contrast enhancement in the thickened appendiceal wall and/or pericecal inflammatory changes, or on the visualization of an abscess, with or without an appendicolith: ${ }^{13}$ one study has described the imaging features of USG and ACT for negative appendectomies in children. ${ }^{8}$ Taylor et a $1{ }^{14}$ concluded that isolated ACT findings of an appendicolith without an inflammatory sign or minimal fat stranding were not sufficient signs for the diagnosis of appendicitis. Trout et al ${ }^{15}$ showed that nonvisualization of the appendix was not related to accurate diagnosis of appendicitis because the smaller volume of intra-abdominal fat in children may contribute to the relatively low rate of normal appendix detection at $\mathrm{ACT} .{ }^{13}$ More recently, Kim et al ${ }^{16}$ reported on some ACT findings that led radiologists to misdiagnosing acute appendicitis. In their study, Kim et al, ${ }^{16}$ found that radiologists frequently misdiagnosed acute appendicitis if an appendicolith or hyperdense materials were observed in the appendix, and the presence of periappendiceal fat inflammation was a key

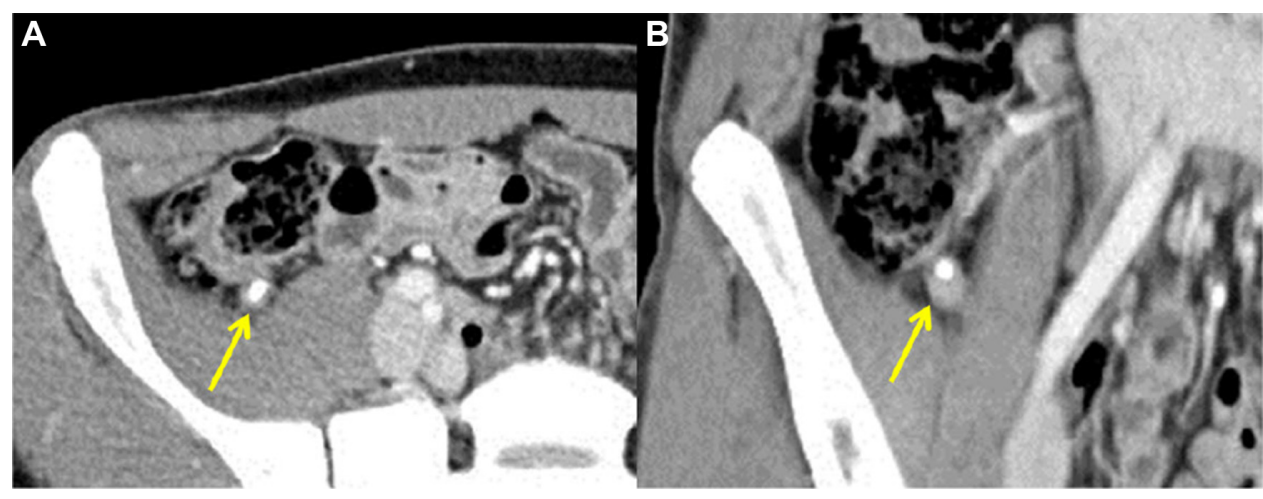

Figure I Preoperative ACT findings for a 16-year-old male patient, who was later demonstrated to have no pathological or diagnostic abnormalities.

Notes: (A) Appendiceal wall thickening and (B) appendicolith are observed (arrows), but there was no associated periappendiceal fat inflammation or fluid collection. Abbreviation: ACT, abdominal computed tomography. 


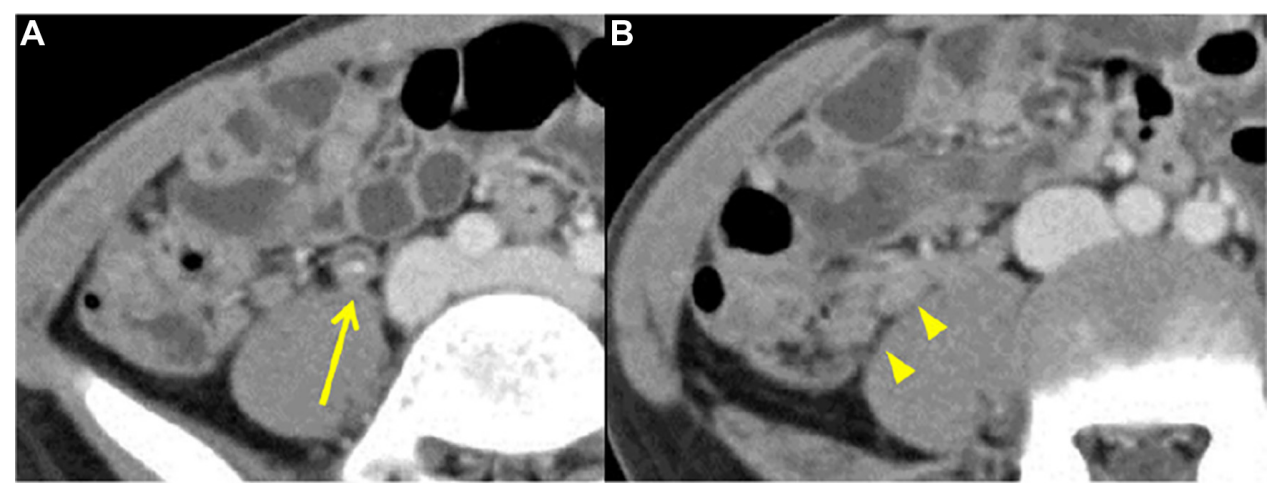

Figure 2 Preoperative ACT findings for a 9-year-old boy, who was later demonstrated to have serosal congestion.

Notes: (A) Appendiceal wall thickening with a small appendicolith (arrow) and (B) adjacent mesenteric lymphadenopathy (arrowheads) are observed, but there was no periappendiceal fat inflammation or fluid collection.

Abbreviation: ACT, abdominal computed tomography.

finding that helped to avoid operating for negative appendicitis in indeterminate cases. However, the studies mentioned herein were just descriptive studies showing the frequency of various findings in negative appendectomy cases; the findings observed were not compared to true appendicitis cases to validate statistical differences. In our study, we measured various factors suggestive of acute appendicitis in a true or negative appendicitis group and validated the statistical differences. In this study, the presence of an appendicolith or intra-appendiceal hyperdense materials was not a determinant factor for diagnosing appendicitis in inconclusive cases, but the presence of periappendiceal fat inflammation was a key determinant. Moreover, appendiceal enlargement, appendiceal wall thickening, and mesenteric lymphadenopathy were also determinants for true or negative appendicitis.

Table 2 Comparison of ACT findings between negative and true appendicitis groups

\begin{tabular}{|c|c|c|c|}
\hline CT findings & $\begin{array}{l}\text { Negative } \\
\text { appendicitis } \\
(\mathrm{N}=10)\end{array}$ & $\begin{array}{l}\text { True } \\
\text { appendicitis } \\
(\mathbf{N}=39)\end{array}$ & $\begin{array}{l}P \text {-value } \\
(\text { OR, 95\% CI) }\end{array}$ \\
\hline $\begin{array}{l}\text { Appendiceal } \\
\text { enlargement }\end{array}$ & I (10\%) & 29 (74.4\%) & $\begin{array}{l}0.000 \\
(26.1,2.92-232.61)\end{array}$ \\
\hline $\begin{array}{l}\text { Appendicolith } \\
\text { or HM }\end{array}$ & $4(40 \%)$ & $8(20.1 \%)$ & $\begin{array}{l}0.233 \\
(0.39,0.09-I .7 I)\end{array}$ \\
\hline $\begin{array}{l}\text { Periappendiceal } \\
\text { fat inflammation }\end{array}$ & I (10\%) & 32 (82.1\%) & $\begin{array}{l}0.000 \\
(41.1,4.46-379.56)\end{array}$ \\
\hline $\begin{array}{l}\text { Periappendiceal } \\
\text { fluid collection }\end{array}$ & $0(0 \%)$ & II (28.2\%) & $\begin{array}{l}0.09 \\
(0.74,0.61-0.89)\end{array}$ \\
\hline $\begin{array}{l}\text { Appendiceal wall } \\
\text { thickening }\end{array}$ & $6(60 \%)$ & $39(100 \%)$ & $\begin{array}{l}0.00 I \\
(7.5,3.56-15.79)\end{array}$ \\
\hline Gas in appendix & $4(40 \%)$ & $9(23.1 \%)$ & $\begin{array}{l}0.422 \\
(0.45,0.11-1.95)\end{array}$ \\
\hline $\begin{array}{l}\text { Mesenteric } \\
\text { lymphadenopathy }\end{array}$ & $6(60 \%)$ & 38 (97.4\%) & $\begin{array}{l}0.004 \\
(25.3,2.40-266.80)\end{array}$ \\
\hline
\end{tabular}

Note: Bold values denote statistical significance.

Abbreviations: $\mathrm{ACT}$, abdominal computed tomography; $\mathrm{Cl}$, confidence interval; $\mathrm{CT}$, computed tomography; HM, hyperdense material; OR, odds ratio.
Therefore, if confronted with an inconclusive radiological report, physicians should consider the presence or absence of these findings as a guide to make a final decision regarding surgical intervention. Using these ACT criteria to diagnose appendicitis, Poortman et $a{ }^{17}$ observed no inconclusive ACT cases when an initial USG was negative or inconclusive. However, the study was conducted in adult patients and therefore those results could not be extrapolated to pediatric patients who have unique anatomic characteristics.

Although we found some determinant findings regarding inconclusive ACT findings, these results should be cautiously interpreted. True appendicitis was diagnosed in 32 of the 33 patients with periappendiceal fat inflammation and in 29 of the 30 patients with an enlarged appendix. Therefore, for cases with inconclusive ACT findings, the presence of periappendiceal fat inflammation or appendiceal enlargement should be interpreted as a positive indicator of true appendicitis. If inconclusive ACT findings were lacking with periappendiceal fat infiltration or appendiceal enlargement, then one should consider the possibility of a negative appendicitis case. However, appendiceal wall thickening or mesenteric lymphadenopathy was present in $60 \%$ of negative appendectomy patients; all four patients who did not have wall thickening and four of the five patients who did not have lymphadenopathy did not have histologically proven appendicitis. Therefore, the absence of these findings has greater clinical implication, indicating negative appendicitis, and clinicians should not be misled to a false-positive diagnosis of appendicitis just with the findings of appendiceal wall thickening or mesenteric lymphadenopathy.

In an effort to reduce radiation hazards while enhancing diagnostic accuracy, various protocols have been implemented for diagnosing pediatric patients with appendicitis. Recently, staged USG and ACT protocols have been used for achieving 
such goals. ${ }^{18,19}$ In this protocol, USG is performed first for patients suspected of having appendicitis, and follow-up CT is recommended if the USG findings were equivocal; the negative appendectomy rate was $8.1 \%$. Recommending ACT as a first-line radiological evaluation is not a key message in our study, and it has already been proven that the increasing utilization of ACT does not decrease the negative appendectomy rate. $^{20,21}$ We also believe in the policy of reducing radiation exposure as much as possible, and the purpose of our study was to provide some clues to help differentiate true and negative appendicitis for patients with inconclusive ACT results. As an alternative to ACT, magnetic resonance imaging (MRI) has been shown to have better diagnostic accuracy without the risk of radiation exposure and might supplant the role of ACT in pediatric appendicitis. ${ }^{22}$ Although we could not use MRI in the diagnostic process due to the limitation of the payment system, further studies are needed to clarify the role of MRI in cases of inconclusive ACT results.

A problem still exists regarding what to do for patients clinically suspected of appendicitis but who have inconclusive ACT findings, suggesting negative appendicitis. In this study group, we had diagnosed patients with appendicitis and performed appendectomies on a clinical basis. Modahl et $\mathrm{al}^{23}$ had reported that leukocytosis and elevated C-reactive protein (CRP) level were indicators of a positive ACT yield. More recently, neutrophil-to-lymphocyte ratio (NLR) has emerged as an alternative marker of systemic inflammation and has demonstrated its utility as a diagnostic marker of childhood appendicitis. ${ }^{24}$ Although we did not assess these laboratory values and follow-up imaging after inconclusive ACT findings in this study and despite acknowledging that the diagnosis of acute appendicitis is not solely dependent upon radiological examination, we would recommend interpretation of these inflammatory markers (leukocytosis, CRP, and NLR), along with short-term imaging and clinical follow-up, rather than surgical exploration. Active clinical observation can increase diagnostic accuracy without increasing morbidity and mortality from appendicitis in suspected cases..$^{25}$ Additional studies are necessitated regarding inflammatory markers and followup imaging with either ACT or USG for patients who have inconclusive ACT findings upon initial examination.

\section{Conclusion}

In summary, we sought to determine some ACT findings that would be suggestive of true or negative appendicitis for pediatric cases with inconclusive ACT results. We found that the presence of either an enlarged appendix of diameter $>8 \mathrm{~mm}$ or periappendiceal fat inflammation strongly correlated with true appendicitis, and the absence of appendiceal wall thickening or mesenteric lymphadenopathy correlated with negative appendicitis. For cases with inconclusive ACT findings for suspected appendicitis, we could further decrease the negative appendectomy rate in children by combining these findings.

\section{Disclosure}

The authors report no conflicts of interest in this work.

\section{References}

1. Korley FK, Pham JC, Kirsch TD. Use of advanced radiology during visits to US emergency departments for injury-related conditions, 1998-2007. JAMA. 2010;304(13):1465-1471.

2. Broder J, Fordham LA, Warshauer DM. Increasing utilization of computed tomography in the pediatric emergency department, 2000-2006. Emerg Radiol. 2007;14(4):227-232.

3. Doria AS. Optimizing the role of imaging in appendicitis. Pediatr Radiol. 2009;39(Suppl 2):S144-S148.

4. Colvin JM, Bachur R, Kharbanda A. The presentation of appendicitis in preadolescent children. Pediatr Emerg Care. 2007;23(12):849-855.

5. Stengel JW, Webb EM, Poder L, Yeh BM, Smith-Bindman R, Coakley FV. Acute appendicitis: clinical outcome in patients with an initial false-positive CT diagnosis. Radiology. 2010;256(1):119-126.

6. Searle AR, Ismail KA, Macgregor D, Hutson JM. Changes in the length and diameter of the normal appendix throughout childhood. $J$ Pediatr Surg. 2013;48(7):1535-1539.

7. Lowe LH, Penney MW, Scheker LE, et al. Appendicolith revealed on CT in children with suspected appendicitis: how specific is it in the diagnosis of appendicitis? AJR Am J Roentgenol. 2000;175(4):981-984.

8. Je BK, Kim SB, Lee SH, Lee KY, Cha SH. Diagnostic value of maximal-outer-diameter and maximal-mural-thickness in use of ultrasound for acute appendicitis in children. World J Gastroenterol. 2009;15(23):2900-2903.

9. Karmazyn B, Werner EA, Rejaie B, Applegate KE. Mesenteric lymph nodes in children: what is normal? Pediatr Radiol. 2005; 35(8):774-777.

10. Morse BC, Roettger RH, Kalbaugh CA, Blackhurst DW, Hines WB Jr. Abdominal CT scanning in reproductive-age women with right lower quadrant abdominal pain: does its use reduce negative appendectomy rates and healthcare costs? Am Surg. 2007;73(6):580-584.

11. Pinto Leite N, Pereira JM, Cunha R, Pinto P, Sirlin C. CT evaluation of appendicitis and its complications: imaging techniques and key diagnostic findings. AJR Am J Roentgenol. 2005;185(2):406-417.

12. Flum DR, Koepsell T. The clinical and economic correlates of misdiagnosed appendicitis: nationwide analysis. Arch Surg. 2002;137(7):799-804

13. Kaiser S, Frenckner B, Jorulf HK. Suspected appendicitis in children: US and CT - a prospective randomized study. Radiology. 2002; 223(3):633-638.

14. Taylor GA, Callahan MJ, Rodriguez D, Smink DS. CT for suspected appendicitis in children: an analysis of diagnostic errors. Pediatr Radiol. 2006;36(4):331-337.

15. Trout AT, Sanchez R, Ladino-Torres MF, Pai DR, Strouse PJ. A critical evaluation of US for the diagnosis of pediatric acute appendicitis in a real-life setting: how can we improve the diagnostic value of sonography? Pediatr Radiol. 2012;42(7):813-823.

16. Kim SH, Choi YH, Kim WS, Cheon JE, Kim IO. Acute appendicitis in children: ultrasound and CT findings in negative appendectomy cases. Pediatr Radiol. 2014;44(10):1243-1251.

17. Poortman P, Oostvogel HJ, Bosma E, et al. Improving diagnosis of acute appendicitis: results of a diagnostic pathway with standard use of ultrasonography followed by selective use of CT. J Am Coll Surg. 2009;208(3):434-441. 
18. Srinivasan A, Servaes S, Peña A, Darge K. Utility of CT after sonography for suspected appendicitis in children: integration of a clinical scoring system with a staged imaging protocol. Emerg Radiol. 2015;22(1):31-42.

19. Krishnamoorthi R, Ramarajan N, Wang NE, et al. Effectiveness of a staged US and CT protocol for the diagnosis of pediatric appendicitis: reducing radiation exposure in the age of ALARA. Radiology. 2011;259(1):231-239.

20. Martin AE, Vollman D, Adler B, Caniano DA. CT scans may not reduce the negative appendectomy rate in children. $J$ Pediatr Surg. 2004;39(6):886-890.

21. Kim Y, Kang G, Moon SB. Increasing utilization of abdominal CT in the Emergency Department of a secondary care center: does it produce better outcomes in caring for pediatric surgical patients? Ann Surg Treat Res. 2014;87(5):239-244.
22. Kulaylat AN, Moore MM, Engbrecht BW, et al. An implemented MRI program to eliminate radiation from the evaluation of pediatric appendicitis. J Pediatr Surg. 2015;50(8):1359-1363.

23. Modahl L, Digumarthy SR, Rhea JT, Conn AK, Saini S, Lee SI. Emergency department abdominal computed tomography for nontraumatic abdominal pain: optimizing utilization. J Am Coll Radiol. 2006;3(11):860-866.

24. Liao W, Zhang J, Zhu Q, et al. Preoperative neutrophil-to-lymphocyte ratio as a new prognostic marker in hepatocellular carcinoma after curative resection. Transl Oncol. 2014;7(2):248-255.

25. Jones PF. Suspected acute appendicitis: trends in management over 30 years. Br J Surg. 2001;88(12):1570-1577.

\section{Publish your work in this journal}

Reports in Medical Imaging is an international, peer-reviewed, open access journal publishing original research, reports, reviews and commentaries on all areas of medical imaging. The manuscript management system is completely online and includes a very quick and fair peer-review system, which is all easy to use.

\section{Dovepress}

Visit http://www.dovepress.com/testimonials.php to read real quotes from published authors. 